\title{
The outburst of Nova CSS 081007:030559+054715 (HV Ceti)
}

\author{
A. P. Beardmore ${ }^{1}$, J. P. Osborne ${ }^{1}$, K. L. Page ${ }^{1}$, P. J. Hakala ${ }^{2}$, G. J. Schwarz ${ }^{3}$, T. Rauch ${ }^{4}$, S. Balman ${ }^{5}$, P. A. Evans ${ }^{1}$, \\ M. R. Goad ${ }^{1}$, J.-U. Ness ${ }^{6}$, S. Starrfield ${ }^{7}$, and R. M. Wagner ${ }^{8}$
}

\author{
${ }^{1}$ Dept. of Physics and Astronomy, University of Leicester, Leicester, LE1 7RH, UK \\ e-mail: ab271@leicester.ac.uk \\ 2 Finnish Centre for Astronomy with ESO, Väisäläntie 20, University of Turku, 21500 Piikkiö, Finland \\ 3 American Astronomical Society, 2000 Florida Avenue, NW, Suite 400, Washington, DC 20009-1231, USA \\ ${ }^{4}$ Institute for Astronomy and Astrophysics, Kepler Center for Astro and Particle Physics, Eberhard Karls University, Sand 1, \\ 72076 Tübingen, Germany \\ 5 Department of Physics, Middle East Technical University, Ankara, Turkey \\ 6 XMM-Newton Science Operations Centre, ESAC, Apartado 78, 28691 Villanueva de la Cañada, Madrid, Spain \\ 7 School of Earth and Space Exploration, Arizona State University, Tempe, AZ 85287-1404, USA \\ 8 Department of Astronomy, The Ohio State University, 140 W. 18th Avenue, Columbus, OH 43210, USA
}

Received 25 May 2012 / Accepted 17 August 2012

\section{ABSTRACT}

\begin{abstract}
Aims. The outburst of nova CSS 081007:030559+054715 (HV Cet) was extensively followed by Swift to study the evolution of its X-ray and UV emission.

Methods. We obtained Swift X-ray and UV observations, at times with high cadence, over an interval of 266 days and analysed the temporal and spectral properties of the object. Optical polarisation measurements were also obtained, but no significant circular polarisation was identified. UV data from GALEX and AAVSO optical data were investigated in addition.

Results. A variable supersoft source was discovered, with a $1.77 \mathrm{~d}$ periodic modulation in its X-ray, UV and optical emission, in phase across all three bands. High cadence observations revealed a declining trend of the X-ray modulation amplitude, while the UV oscillation became stronger over this interval; the UV data also show occasional secondary minima. The X-ray and UV flux declines sharply 150 days after discovery (the date of optical outburst peak is uncertain). While bright, the UV and, to a lesser extent, the $\mathrm{X}$-ray light curves show two cycles of a $\sim 40-50$ day modulation. We believe the $1.77 \mathrm{~d}$ modulation to be orbital, likely caused by structured disc-rim occultation. The X-ray spectra were soft at all times, with negligible flux above $1 \mathrm{keV}$. These spectra were fitted with an NLTE white dwarf model atmosphere of $k T=60-80 \mathrm{eV}$, with no spectral changes related to the $1.77 \mathrm{~d}$ or possible $\sim 40$-day periodicities seen. A Cloudy model demonstrated that it is possible to account for the UV flux through the reprocessing of X-rays. The UV flux is far in excess of the soft X-ray spectral component. We propose that the apparently sub-Eddington white dwarf luminosity is due to permanent obscuration by the accretion disc rim: we see only X-rays scattered by optically thin plasma interior to the disc rim, whereas the UV flux is mostly created by reprocessing in the disk rim interior surface. The in-phase $1.77 \mathrm{~d}$ UV and X-ray modulation is caused by the region in which the gas stream from the secondary raises the disc rim, the maximum X-ray obscuration coinciding with the minimum aspect of the UV reprocessing area. In summary, HV Cet had characteristics of both classical novae and persistent super-soft X-ray sources.
\end{abstract}

Key words. novae, cataclysmic variables - stars: individual: CSS 081007:030559+054715 (HV Cet) - X-rays: stars - ultraviolet: stars

\section{Introduction}

CSS 081007:030559+054715 (named by Kazarovets et al. 2011, as HV Cet) was discovered by the Catalina Real-time Transient Survey (CRTS; Drake et al. 2009) on 2008-Oct-07 (MJD 54746.38112 ) as an object which had brightened by about 4 mag to a $V$-band magnitude of 15.8 over the course of a year (Pejcha et al. 2008), though a gap lasting 181 days $^{1}$ precluded the discovery of the precise outburst time or the rate of rise. The All Sky Automated Survey (Pojmanski 2002) observed the source during the CRTS gap and detected it close to the limiting magnitude of the survey, recording a maximum $V$-band magnitude of 14.3 on 2008-Jul-24 (Prieto et al. 2008). Optical spectroscopy obtained on 2008-Oct-27 and 2008-Oct-30 revealed a multi-peaked $\mathrm{H} \alpha$ emission line profile with radial velocities exceeding $\pm 1500 \mathrm{~km} \mathrm{~s}^{-1}$, as well as strong double-peaked

\footnotetext{
${ }^{1}$ http://nesssi.cacr.caltech.edu/catalina/20081007/ $810071070174103058 \mathrm{p} \cdot \mathrm{html}$
}

[Ne V] emission at $3426 \AA$ (Pejcha et al. 2008; Prieto et al. 2008). This led the authors to suggest the source might be a fast neon nova similar to V4160 Sgr (Williams et al. 1994), which in turn has similarities with V838 Her (Schwarz et al. 2007). The broad hydrogen emission lines, the $>4$ mag increase in brightness and even the pre-outburst variability of 1-2 mag (Drake et al. 2009), similar to those of recurrent novae (Schaefer 2010), all point to this object being a nova, in spite of its unusual Galactic location at $l, b=172.6,-43.7$.

$\mathrm{HV}$ Cet is interesting in that it shares both nova and compact/close binary supersoft source (CBSS) characteristics. Classical novae are binary systems in which a white dwarf (WD) accretes hydrogen-rich material from a companion, usually through an accretion disc, at a rate below $\sim 10^{-8} M_{\odot} \mathrm{yr}^{-1}$. When enough material has been accumulated onto the WD, the deeper accreted layers become degenerate and the temperature and pressure increase sufficiently to trigger a thermonuclear runaway (TNR), resulting in a nova explosion 
(see Bode \& Evans 2008, for a review). While some fraction of the accreted material is ejected with velocities exceeding the escape velocity of the WD (i.e., a few thousand $\mathrm{km} \mathrm{s}^{-1}$ ), the remainder is expected to burn at constant bolometric luminosity (e.g., Gallagher \& Code 1974; Sparks et al. 1976; Prialnik 1986). During this phase the photosphere of the WD shrinks, causing its effective temperature to increase which, once the ejecta have cleared sufficiently, gives rise to strong soft X-ray emission; i.e. the nova becomes a supersoft X-ray source. Nuclear burning continues and the effective temperature slowly increases until the remaining material is consumed, at which point there should be a sharp decline in the X-ray flux from the WD.

Supersoft X-ray sources (SSS) were initially identified by the Einstein Observatory, with subsequent discoveries by ROSAT leading to the recognition that such sources were a new and important class of object (Trümper et al. 1991). It was determined that their observed characteristics could be explained by nuclear burning on the surface of the accreting WD, with typical X-ray luminosities of $\sim 10^{36}-10^{38} \mathrm{erg} \mathrm{s}^{-1}$, up to about the Eddington luminosity for a $1 M_{\odot}$ star (Kahabka \& van den Heuvel 1997). Both transient and persistent SSS are known (see Di Stefano et al. 2010, for a discussion of different SSS populations), with all novae predicted to undergo a supersoft phase during their lifetime. This soft emission was observed in several novae (e.g., Krautter et al. 1996; Drake et al. 2003; Ness et al. 2003; Orio et al. 2002), with the more recent Swift campaigns (Schwarz et al. 2011) providing detailed monitoring of a number of sources, such as RS Oph (Osborne et al. 2011), V2491 Cyg (Page et al. 2010), KT Eri (Bode et al. 2010; Beardmore et al. 2010), Nova LMC 2009a (Bode et al. 2009a,b, and in prep.) and Nova LMC 2012 (Page et al. 2012).

The persistent supersoft sources can be explained by the CBSS model (van den Heuvel et al. 1992; Kahabka \& van den Heuvel 1997), although see Starrfield et al. (2012). In these systems, the accretion rate is sufficiently high (few $\times$ $10^{-7} M_{\odot} \mathrm{yr}^{-1}$ ) that nuclear burning occurs indefinitely. The high accretion rate arises because, unlike in novae, the mass-losing star is likely to be more massive than the WD, resulting in unstable mass transfer on the thermal timescale of the donor star.

The Swift observatory (Gehrels et al. 2004), with its coaligned X-ray (the XRT; Burrows et al. 2005) and UV/optical (the UVOT; Roming et al. 2005) telescopes and rapid response capability, makes it an ideal facility to observe novae. The presence of the $[\mathrm{Ne} \mathrm{V}]$ emission in the optical spectrum from HV Cet, which can be produced by photoionisation processes providing the illuminating photons have energies in excess of $126 \mathrm{eV}$, strongly suggested the underlying nuclear burning WD was hot and would be a soft X-ray source. Hence, a Swift Target of Opportunity (ToO) observation was requested by the Swift Nova-CV group, in order to characterise its X-ray and UV behaviour. Following this initial observation, the source was monitored extensively with Swift. The data reveal a soft, blackbodylike spectrum, variable X-ray and UV light-curves with a $1.77 \mathrm{~d}$ period in both bands, and a slowly declining trend in the soft $\mathrm{X}$-ray and UV flux. The preliminary results were presented by Beardmore et al. (2010) and this paper expands on the initial work done, in particular, parameterising each X-ray spectrum with a model atmosphere, providing the GALEX UV spectrum and investigating the link between the UV and X-ray emission.

\section{Observations and analysis}

Our observations of HV Cet consist of $266 \mathrm{ks}$ of exposure by Swift, spanning 266 days from 2008-Nov-10 to 2009-Aug-03, and Nordic Optical Telescope (NOT) polarisation data obtained on 2009-Feb-11. GALEX (Galaxy Evolution Explorer) also observed HV Cet, both in direct imaging and grism mode (Sect. 2.3) and we additionally make use of photometry obtained from the American Association of Variable Star Observers (AAVSO).

The initial Swift ToO observation took place on 2008-Nov-10, though it was interrupted by observing constraints after only $530 \mathrm{~s}$. It was sufficient, however, to reveal a soft X-ray source in which a total of 75 counts were detected, all below $1 \mathrm{keV}$. The observation was rescheduled on 2008-Nov-14 for a further $3.1 \mathrm{ks}$. These additional data showed a clear signature of a SSS, which could be fitted with a $38 \mathrm{eV}$ blackbody model (Schwarz et al. 2008). The source was also detected by the UVOT at a magnitude of 15.1 in the uvw2 filter (central wavelength of $1928 \AA$ ). The Swift X-ray position, obtained by astrometrically correcting these data using the XRT-UVOT alignment and matching UVOT field sources to the USNO-B1 catalogue (see Goad et al. 2007; Evans et al. 2009), is $\mathrm{RA}(\mathrm{J} 2000)=030558.55, \operatorname{Dec}(\mathrm{J} 2000)=+0547$ 15.4, with an $90 \%$ error radius of 2.0 arcsec, while the UVOT position is $\operatorname{RA}(2000)=030558.53, \operatorname{Dec}(2000)=+054714.7$, with a $1 \sigma$ uncertainty of 0.5 arcsec.

A further ToO request lasted until 2008-Nov-21, with 5-6 ks of data being collected every alternate day, during which time both the XRT and UVOT data showed only slight variability, with a root mean square (rms) amplitude of a few percent; the mean XRT count rate $(0.3-10 \mathrm{keV})$ and UV magnitude at this time were 0.18 count $\mathrm{s}^{-1}$ and 14.9 , respectively.

Following an eight day hiatus, Swift observations resumed, collecting $1-2 \mathrm{ks}$ of data per day from 2008-Nov-30 to 2008-Dec-09. While the data were sparse, a highly variable soft $\mathrm{X}$-ray source was revealed, with an XRT count rate ranging from $0.2-2.2$ count s$^{-1}$, with a periodicity of $1.77 \mathrm{~d}$ (Beardmore et al. 2008). The UVOT uvw2 data also varied, from 14.6-14.0 mag, on the same timescale and in phase with the X-rays (see Sect. 2.1.1). After the discovery of the $1.77 \mathrm{~d}$ periodicity, we obtained two sets of high cadence observations with Swift, aiming for $500 \mathrm{~s}$ of data every Swift orbit (i.e. every $96 \mathrm{~min})^{2}$. The first ran daily from 2008-Dec-12 to 2008-Dec-24 and the second ran for four days a week from 2009-Jan.-18 to 2009-Feb-09, while approximately daily $1 \mathrm{ks}$ observations took place in between the two high cadence campaigns. A return to lower cadence observations ( $\sim 1 \mathrm{ks}$ per day) occurred from 2008-Feb-14 until 2009-Mar.-20, at which point the source was too close to the Sun to be safely observed by Swift. A final dataset was obtained when the source re-emerged from behind the Sun, on 2009-Aug-02/03.

The XRT and UVOT data were processed using the standard Swift software tools (version 3.7, corresponding to HEASoft 6.10) and analysed using the corresponding calibration files (version 11 of the X-ray response matrices). The XRT data were taken in Photon Counting (PC) mode throughout the observing campaign. Standard exposure map corrections were applied at all times to take into account the bad columns present on the XRT CCD. Pile-up occurs when more than one photon provides charge to a pixel (or a directly adjacent pixel) before the CCD is read out. Pile-up is more obviously identified in very soft sources, since there are few counts at higher energies to mask the evidence, and source count rates exceeding $\sim 0.3$ count s ${ }^{-1}$ in PC mode can be affected. Care was taken to exclude the piled-up core of the XRT point spread function (PSF) at these

\footnotetext{
2 Subject to interruptions by the discovery of Gamma-Ray Bursts and other ToO requests.
} 


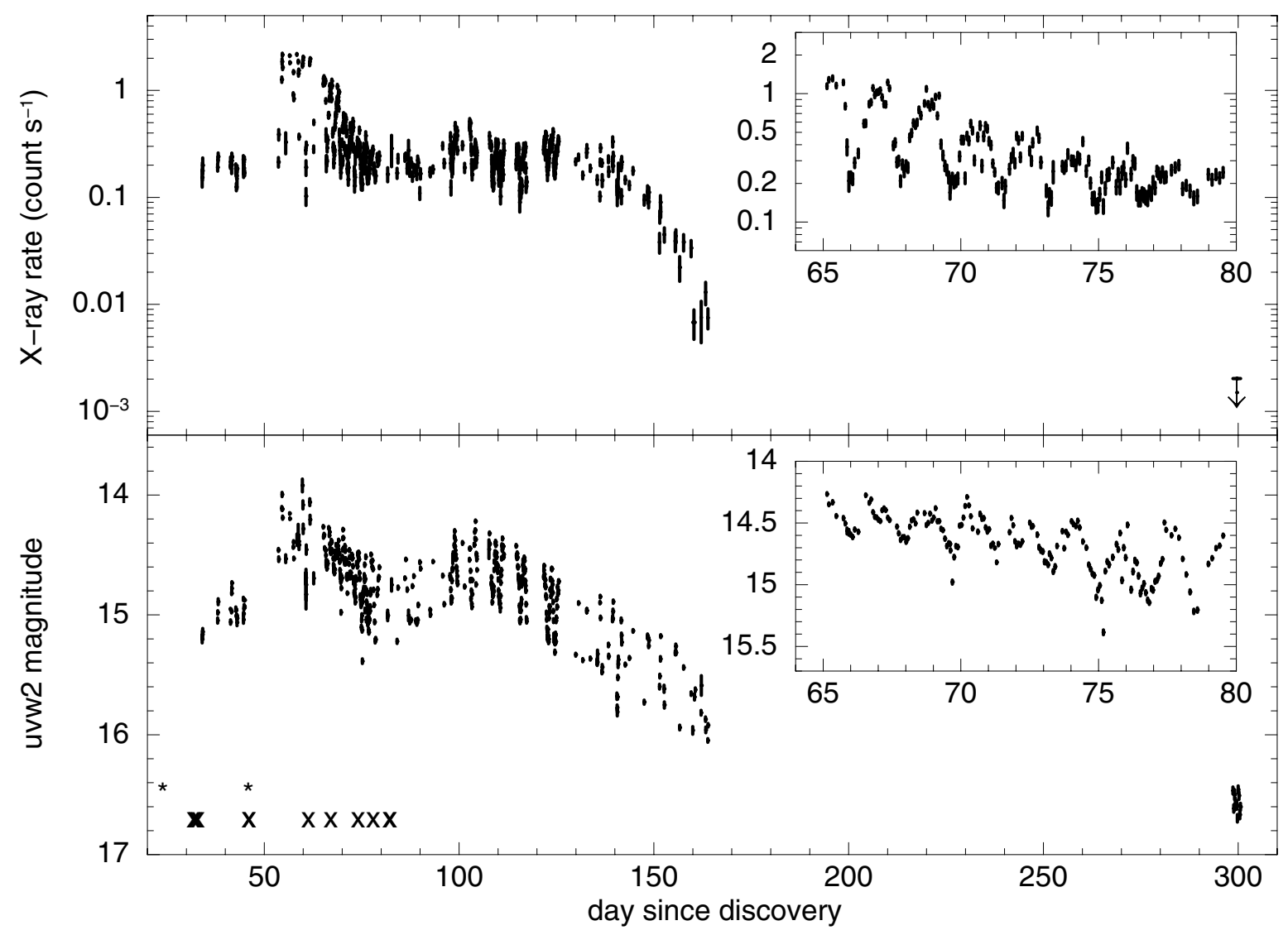

Fig. 1. Swift-XRT 0.3-1.0 keV (top) and UVOT uvw2 (bottom) light-curves of HV Cet. Inset into both panels is a expanded view of the light-curves from day 65 to 80, which covers the first high cadence campaign. The X-ray count rate is plotted with a logarithmic scale for ease of comparison with the UV magnitudes. The discovery date is taken as 2008-Oct-07 at 09:08:49 UTC. In the lower panel, * and $\times$ symbols mark the times of the GALEX imaging and grism observations respectively.

times by using an annular extraction region and correcting for the PSF losses. In order to minimise pile-up issues further, only grade zero (single pixel) events were used when extracting the $\mathrm{X}$-ray spectra. The UVOT data were collected with the uvw2 filter and photometry was performed on the UVOT photometric system described in Poole et al. (2008). There is an uncertainty of 0.03 mag on the $u v w 2$ zero-point which has not been included in the plotted error bars.

\subsection{Light-curves}

The Swift X-ray and UV light-curves for the observing campaign are shown in Fig. 1. The zero time corresponds to 2008-Oct-07 at 09:08:49, the time of discovery ${ }^{3}$, not the time of the optical maximum, since this is uncertain ${ }^{4}$. The $\mathrm{X}$-ray count rates are plotted with a logarithmic axis, in order to facilitate a comparison with the UV magnitudes. However, note that the X-ray data cover a much larger range than the UV (more than a factor of 100 for the XRT, compared to a factor of $\sim 6.3$ - or two magnitudes for the UVOT, when comparing the data prior to day 165). The source only shows significant X-ray flux below $1.0 \mathrm{keV}$, with the count rate in the $1.0-10 \mathrm{keV}$ band being $<3 \times 10^{-3}$ count $\mathrm{s}^{-1}$ at all times.

\footnotetext{
3 http://nesssi.cacr.caltech.edu/catalina/Oct08ns. html\#table61

${ }^{4}$ Neither WASP (Wide Angle Search for Planets) nor SMEI (Solar Mass Ejection Imager) observations further constrain the outburst time.
}

As the inset panels show, there is a clear periodicity in both the X-ray and UV data, with a timescale of $1.77 \mathrm{~d}$ (Osborne et al. 2009). More details are given in Sect. 2.1.1.

During the first high cadence campaign (shown in the insets in Fig. 1), the modulation seen in the X-ray band exhibited a trend of declining maxima. The minimum of the oscillation fades only slightly from $\sim 0.20$ count s $^{-1}$ to 0.14 count $^{-1}$, whereas the maximum decreases by a larger factor, from about 1.4 count s$^{-1}$ to 0.3 count s$^{-1}$; the amplitude of the X-ray $1.77 \mathrm{~d}$ periodicity therefore becomes smaller. The UVOT, on the other hand, shows the opposite effect, with the amplitude of the modulation increasing over this same time interval. By the end of this interval, the amplitudes of the X-ray and UV modulations are comparable. Occasional secondary minima were seen in the UV light-curve during times of the $1.77 \mathrm{~d}$ cycle maxima (e.g. day 66.9 and 72.1).

While the emission in both bands fades between day $\sim 55-80$, the UV source then rebrightens until around day 110 , before starting a slow decline. The X-ray data, on the other hand, do not rebrighten as strongly as the UV and only start to fade significantly after day 140 . These results seem to point to an underlying long-term variation, visible in both the X-ray and UV data, which is also discussed in Sect. 2.1.1.

The final observation was obtained on day 300 . At this time, the UV source was still detected, at a magnitude of 16.6 in the uvw2 band, showing that the source had continued to fade, although less rapidly than was implied by extrapolating the data between days 110 and 165 . The X-ray source was no longer 

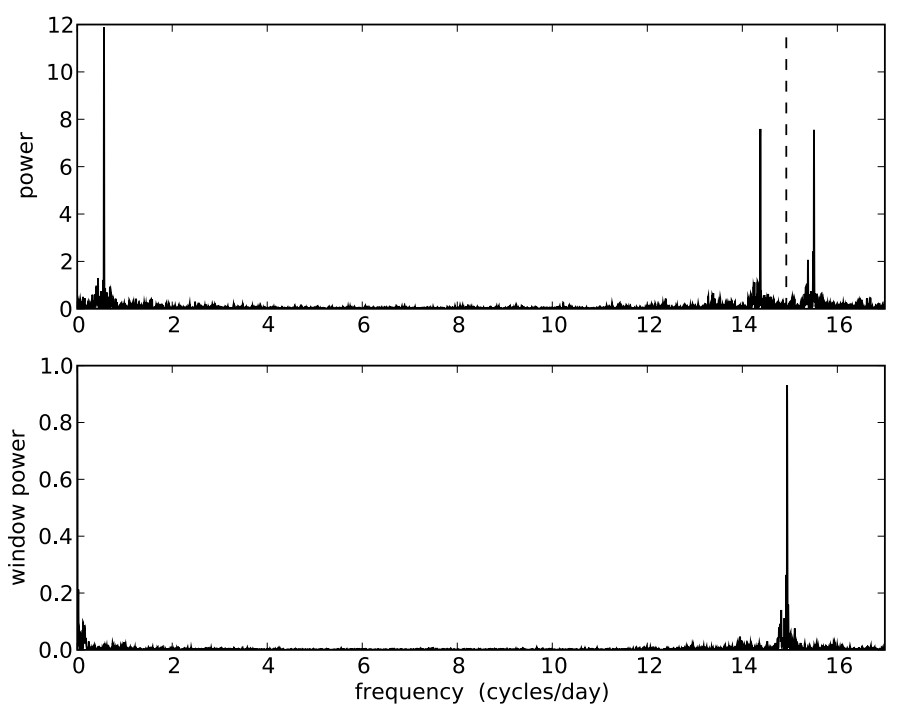

Fig. 2. Lomb-Scargle periodogram of the Swift-UVOT uvw2 data (top), after detrending with a 9 th order polynomial. The $1.77 \mathrm{~d}$ modulation is clearly seen at a frequency of 0.564 cycle day ${ }^{-1}$, along with its corresponding aliases with Swift's orbital period (dashed line) caused by the window function of the data (bottom).

visible, however, with the $6.3 \mathrm{ks}$ of data collected providing an upper limit of $2.0 \times 10^{-3}$ count s$^{-1}$

The earlier ROSAT-PSPC All Sky Survey (data collected in July 1990) did not detect a source at the position of HV Cet to an XRT-equivalent upper limit of $1.6 \times 10^{-4}$ count s $^{-1}$.

\subsubsection{Period search}

There is a clear $1.77 \mathrm{~d}$ modulation detected in the data, both in the X-ray and UV. Figure 2 shows a Lomb-Scargle periodogram of the UV light-curve from day 30 to 165 , following detrending using a ninth order polynomial. A coherent $1.77 \mathrm{~d}$ periodicity is visible at a frequency of 0.564 cycle day $^{-1}$.

As described in Beardmore et al. (2010), and following Sterken (2005), an ephemeris for the times of the UV flux minima $\left(T_{\min }\right)$ was derived using the $u v w 2$ data obtained during the high-cadence campaigns. The uvw2 times were heliocentrically corrected, then the data were fitted for $\sim 1-1.5$ days around the minima with a model consisting of a straight line plus a Gaussian, where the centroid of the latter was used to determine the times of the minima. 18 well-sampled minima were considered and typical uncertainties of 0.07 days in the derived minima times were estimated. From the times of the minima the following ephemeris was obtained:

$$
T_{\min }=\text { HJD } 2454812.9906(0.0198)+1.7718(0.0010) \times E
$$

where $E$ is the cycle count and HJD is the Heliocentric Julian day. The time is in Terrestrial Time (TT) where $\mathrm{TT}=\mathrm{UTC}+65.18 \mathrm{~s}$. The terms in parentheses are $1 \sigma$ error estimates.

When the Swift UV and X-ray data are folded using this ephemeris, the primary UV and X-ray flux minima are found to be in phase (Fig. 3). Fitting the folded X-ray light-curve with the ephemeris derived from the UVOT data, the maximum allowed delay between the X-ray and UV bands is $0.02 P_{\text {orb }}(90 \%$ confidence). This consistency in phase is also true for the softer (0.3-0.45 keV) and harder $(0.45-1 \mathrm{keV}) \mathrm{X}$-ray bands, with no sign of the $1.77 \mathrm{~d}$ oscillation in the hardness ratio (computed as

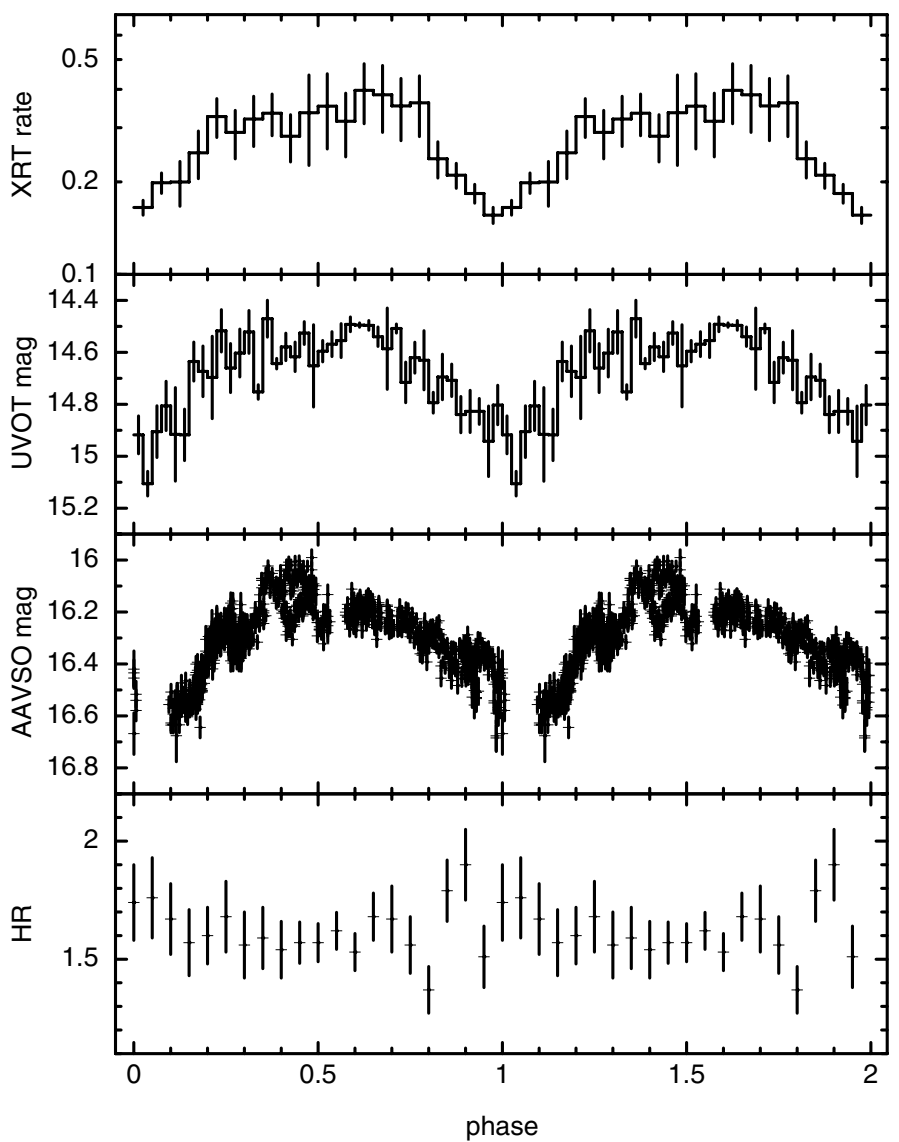

Fig. 3. From top to bottom: the XRT, UVOT, AAVSO data and X-ray hardness ratio $(0.45-1 / 0.3-0.45 \mathrm{keV})$ each folded on the $1.77 \mathrm{~d}$ ephemeris given in the text. All three light-curve datasets are in phase with each other and cover the same time interval of days 71-84 (defined by the availability of AAVSO unfiltered data). The Swift XRT and UVOT datasets have been averaged into 20 and 40 phase bins per cycle respectively, with the uncertainties being the standard errors on the mean. The X-ray hardness ratio shows no significant variation during the $1.77 \mathrm{~d}$ interval.

$0.45-1 / 0.3-0.45 \mathrm{keV}$ ), with a $90 \%$ upper limit of $3.4 \%$ on the amplitude.

Ground-based optical data were also made available by the AAVSO. These data (from day 68.1 to 84.8) also confirm the $1.77 \mathrm{~d}$ period (Templeton et al. 2010). We find the optical data in phase with the X-ray and UV datasets presented here, as shown in Fig. 3, contrary to the report of Templeton et al. (2010); they appear to have performed an incorrect epoch conversion from MJD to HJD.

CCD photometry taken by Frans Nieuenhout (priv. comm.), after the end of the AAVSO data spanning days 94-134, on his $0.4 \mathrm{~m}$ Meade in The Netherlands, have Johnson $V$ magnitudes 16.4-16.7, consistent with the range seen in the AAVSO dataset.

Figure 4 shows individual cycles of the XRT and UVOT data obtained during the first high-cadence monitoring campaign (days 65-80). Phases were determined from the ephemeris and each panel shows the same cycle repeated. The third and fourth UVOT panels most clearly show secondary minima.

While the $1.77 \mathrm{~d}$ periodicity dominates the light-curves there is also possibly a longer timescale modulation of between 40 and 50 days visible, which is particularly noticeable in the UVOT light-curve. At best, however, there were two minima and two maxima of the modulation before the source became too close 


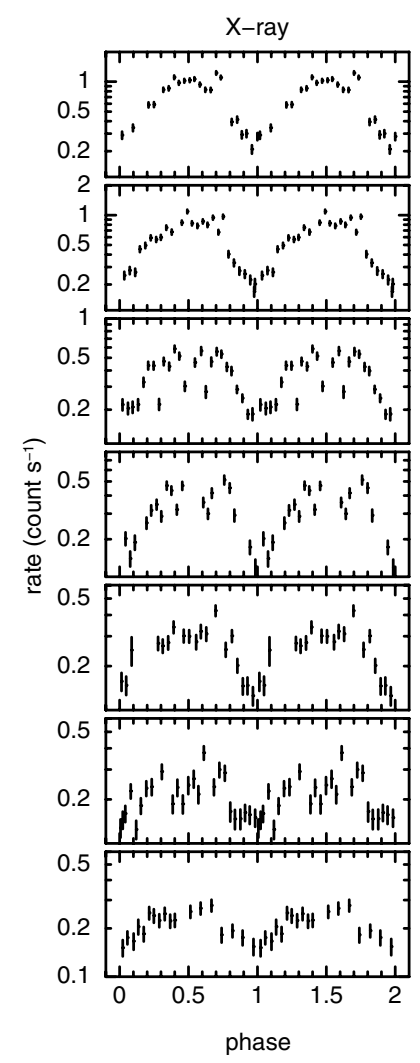

Fig. 4. Repeated single cycles of the $1.77 \mathrm{~d}$ modulation. The Swift XRT and UVOT data are from the first high-cadence monitoring campaign (days 65-80, shown in the insets of Fig. 1).

to the Sun for Swift to follow; it is impossible to know whether this is a periodic phenomenon.

Both RS Oph (Osborne et al. 2011) and KT Eri (Beardmore et al. 2010) have been found to show $\sim 35$-s periods in their X-ray data, with fractional modulation amplitudes of up to 10 and $7 \%$ respectively. HV Cet, however, shows no such significant shortterm modulation, with a $90 \%$ upper limit of $2 \%$ on the amplitude of any modulation around $35 \mathrm{~s}$.

\subsection{Spectra}

Beardmore et al. (2010) presented an average spectrum for HV Cet, finding a very soft, blackbody-like source. Here we investigate whether there is any spectral evolution during the observations.

A hardness ratio (HR; hard band: $0.45-1 \mathrm{keV}$; soft band: $0.3-0.45 \mathrm{keV}$ ) was created ${ }^{5}$ to give an initial overview of any spectral changes occurring during the observation. As the second panel of Fig. 5 shows, overall the hardness ratio remains close to constant for much of the time covered, although there appears to be a substantial hardening and subsequent re-softening of the data between $\sim$ day 110 and 135 . This corresponds to the interval over which the XRT light-curve shows a slight recovery in count rate (or, at least, does not fade in the same way as the UV). As mentioned above, phase-folding the hardness ratio reveals no significant modulation over the $1.77 \mathrm{~d}$ period.

Spectra were then extracted for each individual Observation ID, which typically last up to a day, though can be as short as 500 s. Fig. 6 shows a small sample of the

\footnotetext{
${ }^{5}$ Using the online facility at the UKSSDC (Evans et al. 2009): http://www.swift.ac.uk/user_objects/
}

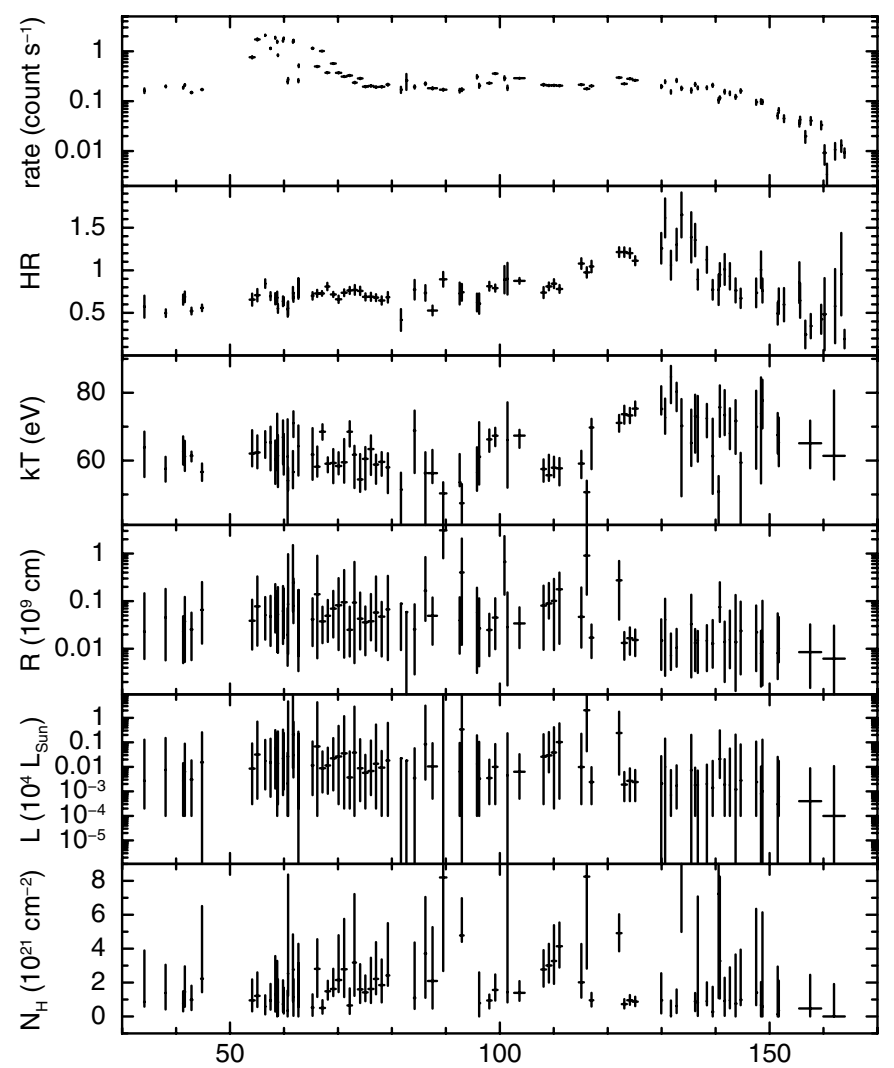

day since discovery

Fig. 5. Swift-XRT light-curve, hardness ratio $(0.45-1 \mathrm{keV} / 0.3-$ $0.45 \mathrm{keV}$ ) and fits to the spectra using an NLTE atmosphere model; the distance to HV Cet is taken to be $2.5 \mathrm{kpc}$ when calculating the radius and luminosity. In each case there is a single data point per Observation ID.

spectra obtained, covering the whole of the observing campaign. The spectrum in black is from the first observation of the source (day 34.1), while the red spectrum was obtained at the peak of the light-curve (day 60.7). The blue spectrum (day 81.7) corresponds to the point at which the source stopped fading and started to rebrighten slightly, while the magenta spectrum covers day 132.8, around which time the temperature of the atmosphere model appears to reach a peak; there is excess emission seen between $\sim 0.6$ and $0.7 \mathrm{keV}$ in this spectrum, which could indicate the presence of $\mathrm{H}$-like and He-like oxygen emission lines. The cyan data were taken at the start of the final decline in X-rays (day 140.6), while the orange spectrum corresponds to days 160-164, covering the final detections of the X-ray source.

The spectra were fitted with a model comprising a planeparallel, static, non-local thermal equilibrium (NLTE) atmosphere component (Rauch 2003; Rauch et al. 2010), a singletemperature optically-thin component (to parameterise the few counts at higher energies; this component was not strongly significant) and a freely varying absorbing column. Since HV Cet is thought to be a neon-rich nova, TMAP model grid $011^{6}$ was used. This model atmosphere grid was calculated for

${ }^{6}$ http://astro.uni-tuebingen.de/ rauch/TMAF/flux HHeCNONeMgSiS_gen.html In the framework of the Virtual Observatory (VO; http://www.ivoa.net), these spectral energy distributions (SEDs, $\lambda-F_{\lambda}$ ) are available in VO compliant form via the VO service TheoSSA (http://vo.ari.uni-heidelberg. de/ssatr-0.01/TrSpectra.jsp?) provided by the German Astrophysical Virtual Observatory (GAVO; http: //www . g-vo.org). 


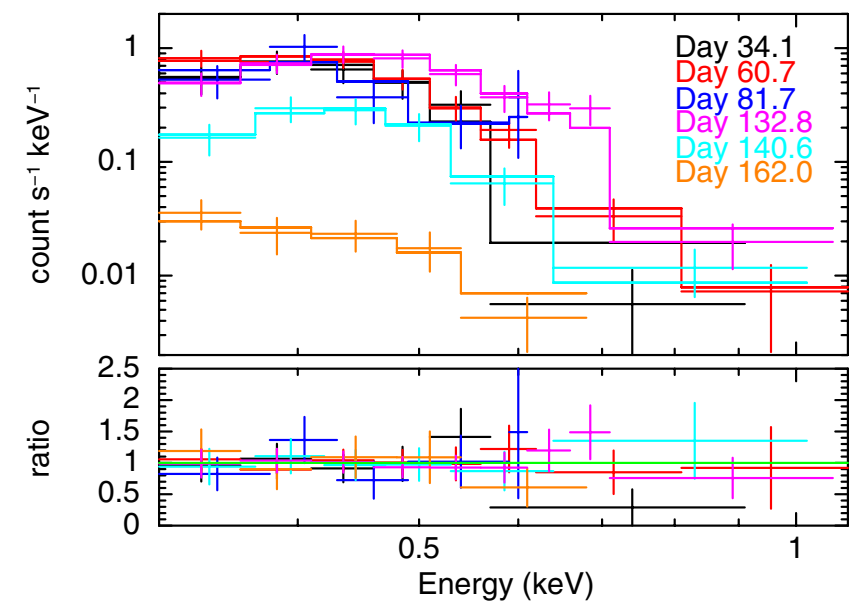

Fig. 6. A sample of Swift-XRT spectra covering the observing campaign, fitted with the atmosphere model described in the text.

temperatures of $(4.5-10.5) \times 10^{5} \mathrm{~K}$, in steps of $10^{4} \mathrm{~K}$. The parameters determined from this spectral fitting are shown in Fig. 5. A distance estimate of $2.5 \mathrm{kpc}$ (Schwarz et al. 2008) was used to calculate the radius and luminosity; their assumptions lead to a distance range of $2.5-10 \mathrm{kpc}$. The radius shown in the figure can be rescaled as $R \propto D / 2.5$, where $D$ is the distance in kpc. The luminosities are plotted in units of $10^{4} L_{\odot}$; the values measured are $\sim 10^{35-36} \mathrm{erg} \mathrm{s}^{-1}$, at the lower end of the range typical for SSS; if the distance were taken to be $10 \mathrm{kpc}$, the luminosities would be a factor of 16 higher. For comparison, the Eddington Luminosity, $L_{\mathrm{Edd}}=3.3 \times 10^{4}\left(M / M_{\odot}\right) L_{\odot}=$ $1.38 \times 10^{38}\left(M / M_{\odot}\right) \mathrm{erg} \mathrm{s}^{-1}$ (assuming solar abundance ratios).

All the spectra are soft, with almost all the photons being detected below $1 \mathrm{keV}$, even as the X-ray emission faded, and little variation in the temperature of the soft component was found, except for the increase between day 110 and 130, from $k T \sim 60$ $80 \mathrm{eV}$. Different TMAP grids were tested, and all showed this increase; in fact, the grid 011 data presented here revealed the most conservative rise in temperature. The effective radius and therefore luminosity are also close to constant, although they may be starting to decrease after about day 120, the combination of this and the falling temperature after day 130 causing the final decay of the XRT count rate. Preliminary analysis of the day 72 Chandra data by Nelson \& Orio (2009) leads to similar estimates for the temperature of the model atmosphere and its absorbing column.

Phase-resolved spectra were also extracted, corresponding to the peak (phases 0.4-0.6) and minimum (phases 0.9-1.1) of the $1.77 \mathrm{~d}$ modulation; only times when the spectra would not be piled-up were used for simplicity. The temperatures of these spectra were found to be consistent: $k T_{\min }=56 \pm 1 \mathrm{eV}$ and $k T_{\max }=57 \pm 1 \mathrm{eV}$, as suggested by the lack of variation in the hardness ratio.

Although the NLTE model atmospheres used do not predict that emission lines will occur, Nelson \& Orio (2009) report the presence of H-like nitrogen Ly $\alpha(0.50 \mathrm{keV})$ and He-like oxygen $(0.57 \mathrm{keV}$ ) emission lines on 2008-Dec-18 (day 72 after discovery). Such lines are not significantly produced by the optically thin component $(k T \sim 2 \mathrm{keV})$ which we used to account for the few counts above $1 \mathrm{keV}$. We wondered whether the excursion to higher temperatures after day 110 might be due to a variation in the oxygen emission line spectrum; fixing the WD temperature to be $60 \mathrm{eV}$, the day 132.8 spectrum (magenta line in Fig. 6) requires an oxygen line ratio temperature of $k T=0.19_{-0.04}^{+0.09} \mathrm{keV}$ assuming collisional ionisation (as is reasonable given that the line energies are above that of the continuum). There appears to be no reason to expect such a pulse of line emission, whereas a shrinking and heating of the nova remnant photosphere is a prediction of the nova model (Iben 1982; MacDonald et al. 1985). Therefore we believe the temperature evolution shown in Fig. 5 reflects the behaviour of the WD.

\subsubsection{Spectral energy distributions}

Since Swift collects UVOT and XRT data simultaneously, spectral energy distribution (SED) fits can easily be performed; to enable this, the TMAP grids were extended down to $\sim 1.9 \mathrm{eV}$ (6530 $\AA$ ). The model used was the same as for the XRT-only fits, with the addition of the ZDUST command within XSPEC, which accounts for the UV extinction, based on Pei (1992). $E(B-V)$ cannot be constrained from a single UV point, and was therefore set to be 0.146 using the Schlegel et al. (1998) extinction maps for the direction of HV Cet (Schwarz et al. 2011).

While this WD atmosphere model appears to account for both the X-ray and UV emission, the luminosities suggested by this single component are high $\left(\sim 10^{40} \mathrm{erg} \mathrm{s}^{-1}\right.$, equivalent to $\left.\sim 100 L_{\mathrm{Edd}}\right)$. A more physically plausible explanation is that the $\mathrm{UV}$ emission is formed through the reprocessing of X-rays. To determine whether this is feasible - i.e., if sufficient UV flux can be generated from the reprocessed X-ray spectrum - the photoionisation code Cloudy (version 08; Ferland et al. 1998, and references therein) was used. Typical values of the filling factor (0.1; assumed to be constant throughout the shell) and the hydrogen density $\left(10^{7} \mathrm{~cm}^{-3}\right.$ at the inner radius, decreasing as $\left.r^{-3}\right)$ were taken (e.g. Schwarz et al. 1997; Schwarz 2002; Schwarz et al. 2007) with the ejecta assumed to be spherically symmetric. The input spectrum was taken to be a BB (temperature stepped between $\sim 3.5 \times 10^{5}$ and $1.2 \times 10^{6} \mathrm{~K}=\sim 30-100 \mathrm{eV}$ to cover the best-fit temperatures found for the X-ray spectra), with a luminosity of $4 \times 10^{35} \mathrm{erg} \mathrm{s}^{-1}$ (see Fig. 5). The radius of the ejecta was also stepped through a grid from $6.5 \times 10^{14}-6.5 \times 10^{15} \mathrm{~cm}$; this was estimated by taking minimum and maximum expansion velocities of $\sim 500$ and $5000 \mathrm{~km} \mathrm{~s}^{-1}$ and assuming these remained constant for 150 days. The results were written out to XSPEC-friendly models using the punch xspec command described by Porter et al. (2006); the transmitted spectrum was most of interest, since this accounts for both the attenuated incident continuum and any diffuse emission re-emitted by the cloud in the direction of the observer. These models were then renormalised to fit the X-ray data and the predicted UV emission compared with the measured UVOT flux at $1928 \AA(6.4 \mathrm{eV})$. This showed that the predicted reprocessed emission can easily account for the flux observed. This was found to be the case for a range of radii and altering the filling factor or hydrogen density would lead to additional degrees of freedom and further combinations of parameter values able to produce the measured UV flux. Without the inclusion of line ratios obtained from optical spectra, no further constraints can be placed. However, it seems clear that the UV flux can be accounted for by reprocessed X-ray emission and a WD atmosphere origin for this flux is not required.

\subsection{GALEX observations}

HV Cet was observed by the GALEX satellite as part of a discretionary observing time (DOT) request. While GALEX was primarily an imaging instrument that produced simultaneous 
far-UV (FUV; 1350-1750 ̊) and near-UV (NUV; 1750$2800 \AA$ ) images, a grism could be added to the light path to provide low resolution $(R=100-300)$, slitless spectroscopy (Morrissey et al. 2007). GALEX observations of novae are sparse because its $1.2^{\circ}$ field of view and sensitive UV detectors made observations in the Galactic plane, where most novae are found, impossible for all but a few sources. HV Cet is positioned well outside the Galactic plane and the surrounding field was observed twice before outburst as part of the GALEX AIS survey. In addition to these two quiescent observations of HV Cet, the DOT program consisted of 11 grism exposures and two direct images obtained between 2008-Oct-31 and 2008-Dec-28. Table 1 provides the observational details of each exposure, the orbital phase as determined from the ephemeris and either the photometry (direct imaging) or integrated flux (grism spectra) for each UV band.

The observations sample all phases of the derived orbital period and cover the the range from $\sim 32-82$ days after discovery; the times are marked in the lower panel of Fig. 1. Five grism observations were obtained prior to the tremendous increase in the X-ray and UV light curves observed 50 days after discovery, with the sixth grism exposure taken very close to the X-ray and UV maximum as measured by Swift. The final five grism observations were taken during the slow decline.

The GALEX spectra are all remarkably similar with emission lines superimposed on blue continua; Fig. 7 shows the coadded spectrum. Strong emission from He II (1640 ̊) is clearly seen with weaker emission from N IV] (1486 ̊) and C IV (1550 $\AA$ ). Given the strong neon emission observed in the optical spectra at this time (Prieto et al. 2008), the feature around $1590 \AA$ is likely a blend of [Ne V] (1575 $\AA$ ) and [Ne IV] (1602 $\AA$ ). In addition, there is a weak line at $2629 \AA$, corresponding to [Mg VII]; another [Mg VII] line occurs at $2510 \AA$ and may also be faintly detected.

A weak $2175 \AA$ feature is also visible in all individual GALEX spectra and the co-added spectrum. The dip can be removed with $E(B-V)=0.15 \pm 0.05$ mag and a standard Galactic extinction curve. This is consistent with the $E(B-V)=0.15$ derived by Schwarz et al. (2008) based on HV Cet's location in the Schlegel et al. (1998) extinction maps. The observed $1400-2800 \AA$ luminosity is $1 \times 10^{34} \mathrm{erg} \mathrm{s}^{-1}$ for $D=2.5 \mathrm{kpc}$.

The GALEX spectra can be co-added because there is surprisingly little relative variation between the individual spectral observations, regardless of HV Cet's orbital phase or time obtained during the bright outburst. The mean FUV to NUV integrated flux ratio of the eleven grism observations was $0.79 \pm 0.07$. Figure 8 shows the individual FUV/NUV flux ratios as a function of orbital phase. The dotted and dashed lines provide the mean and standard deviation while the grism observation number as given in Table 1 is provided next to each data point. Observation numbers $1-5$ were obtained prior to the bright outburst, while numbers 6-11 were taken at maximum and during the subsequent decline.

The consistency of the UV spectra with respect to the orbital phase matches the same trend in the X-ray hardness ratio (see the bottom panel of Fig. 3) and supports the conclusion that the X-ray and UV emission arises from a common source. The spectral data show that the variability in the X-ray and UV light curves cannot be due to temperature changes.

\subsection{Limits on optical polarisation}

AM Her (orbital period of 0.13 day; Stockman et al. 1977; Hearn \& Richardson 1977) is the prototypical polar system, whereby

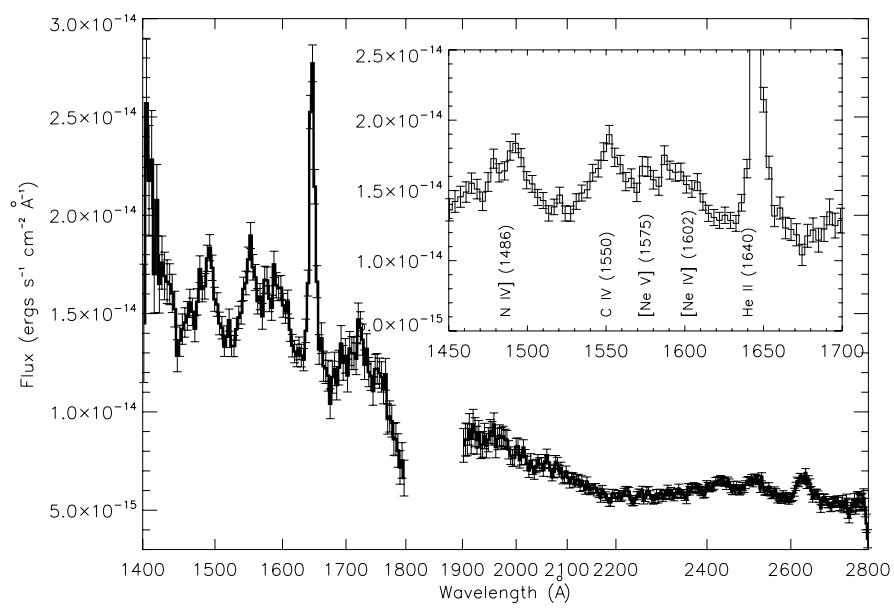

Fig. 7. Co-added spectrum of all the HV Cet GALEX DOT grism observations. Potential emission line identifications in the FUV are given in the inset.

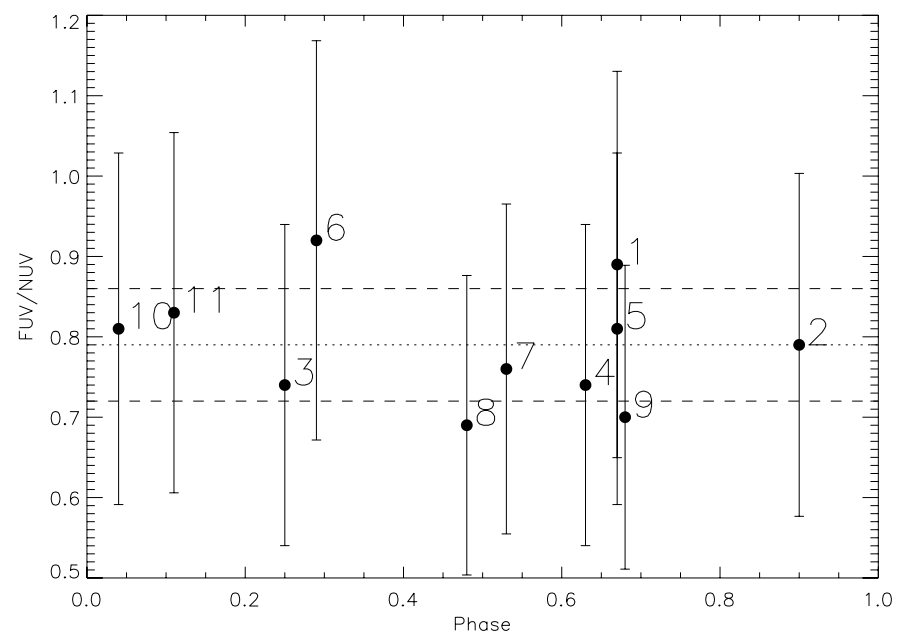

Fig. 8. Ratio of individual FUV/NUV integrated fluxes phased to the derived orbital period. The dotted and dashed lines give the mean and standard deviation of the eleven spectra. Grism observation numbers are given next to each data point. The constant UV "colour" is consistent with the constant hardness ratio in the Swift data.

the strong magnetic field of the WD prevents the inflowing material from forming an accretion disc; instead, the matter spirals along the magnetic field lines, emitting cyclotron radiation and forming hot spots near the magnetic poles of the object as the accretion column impacts the surface. The magnetic field in a polar is strong enough to synchronise the spin periods of the stars with the orbital period of the system. However, a nova explosion can disrupt such synchronicity by a small amount $(<1 \%$; Hellier 2001). V1500 Cyg (Nova Cyg 1975) is an example of this, with optical polarisation measurements taken many years after the nova outburst found to have a period $1.8 \%$ shorter than the orbital period (Stockman et al. 1988). In polars, the soft $\mathrm{X}$-ray emission is eclipsed whenever the X-ray emitting hot spot is occulted by the body of the rotating WD, leading to a similar light-curve morphology as observed in HV Cet (e.g. Hellier 2001). Since the X-ray emission of polars is caused by the accretion column and its subsequent impact, the system becomes $\mathrm{X}$-ray fainter when no matter is being accreted.

Polars are so called because their light is both linearly and circularly polarised. The light-curve of HV Cet shows similarities to that of AM Her, so, to investigate the possibility of this 
Table 1. GALEX observation log.

\begin{tabular}{lccccccc}
\hline \hline Num. & $\begin{array}{c}\text { Obs. date } \\
\text { (UT) }\end{array}$ & $\begin{array}{c}\text { HJD } \\
(\mathrm{d})\end{array}$ & $\begin{array}{c}\text { Time }^{a} \\
(\mathrm{~d})\end{array}$ & $\begin{array}{c}\text { Exp. } \\
(\mathrm{s})\end{array}$ & Phase & FUV $^{b}$ & NUV $^{b}$ \\
\hline & $2003-11-2910: 47: 38$ & 52972.955 & $\begin{array}{c}-1773.93 \\
\text { Direct }\end{array}$ & 110 & 0.49 & $19.71 \pm 0.18$ & $19.40 \pm 0.10$ \\
1 & $2006-11-2011: 23: 41$ & 54059.980 & -686.90 & 104 & 0.00 & $19.45 \pm 0.18$ & $19.41 \pm 0.11$ \\
2 & $2008-10-3120: 29: 17$ & 54771.359 & 24.47 & 1664 & 0.50 & $16.231 \pm 0.008$ & $16.091 \pm 0.005$ \\
3 & $2008-11-2218: 29: 28$ & 54793.276 & 46.39 & 1456 & 0.87 & $16.645 \pm 0.008$ & $16.526 \pm 0.006$ \\
\hline & & \multicolumn{7}{c}{ Grism } & & & \\
1 & $2008-11-08 ~ 05: 59: 56$ & 54778.756 & 31.87 & 880 & 0.68 & $4.50 \times 10^{-12}$ & $5.07 \times 10^{-12}$ \\
2 & $2008-11-0815: 51: 52$ & 54779.167 & 32.28 & 1358 & 0.91 & $3.11 \times 10^{-12}$ & $3.94 \times 10^{-12}$ \\
3 & $2008-11-0906: 39: 46$ & 54779.783 & 32.89 & 960 & 0.26 & $3.56 \times 10^{-12}$ & $4.81 \times 10^{-12}$ \\
4 & $2008-11-2208: 35: 43$ & 54792.864 & 45.98 & 1560 & 0.64 & $4.38 \times 10^{-12}$ & $5.90 \times 10^{-12}$ \\
5 & $2008-11-2210: 14: 17$ & 54792.932 & 46.04 & 1560 & 0.68 & $4.44 \times 10^{-12}$ & $5.46 \times 10^{-12}$ \\
6 & $2008-12-0716: 37: 36$ & 54808.198 & 61.31 & 1678 & 0.29 & $8.19 \times 10^{-12}$ & $8.92 \times 10^{-12}$ \\
7 & $2008-12-1310: 38: 50$ & 54813.948 & 67.06 & 1680 & 0.54 & $4.92 \times 10^{-12}$ & $6.50 \times 10^{-12}$ \\
8 & $2008-12-2010: 14: 19$ & 54820.931 & 74.04 & 1620 & 0.48 & $4.33 \times 10^{-12}$ & $6.30 \times 10^{-12}$ \\
9 & $2008-12-2407: 54: 47$ & 54824.834 & 77.95 & 1480 & 0.68 & $3.74 \times 10^{-12}$ & $5.33 \times 10^{-12}$ \\
10 & $2008-12-2812: 12: 15$ & 54829.012 & 82.13 & 1220 & 0.04 & $3.23 \times 10^{-12}$ & $3.96 \times 10^{-12}$ \\
11 & $2008-12-2815: 29: 37$ & 54829.149 & 82.26 & 1220 & 0.12 & $2.69 \times 10^{-12}$ & $3.22 \times 10^{-12}$ \\
\hline \multicolumn{7}{c}{}
\end{tabular}

Notes. The integrated fluxes are over the ranges 1450-1800 ̊ (FUV) and 1900-2800 ̊ (NUV). ${ }^{(a)}$ Day since discovery, see Fig. 1; 2400000 has been subtracted from the actual HJD value in each case. ${ }^{(b)}$ Units of magnitudes for the direct imaging and erg $\mathrm{cm}^{-2} \mathrm{~s}^{-1}$ for the grism spectra.

source also being a polar, we obtained circular polarisation measurements with the NOT on 2009-Feb-11 (127 days after discovery), close to the maximum of the $1.77 \mathrm{~d}$ modulation; this is the time at which the emitting region is most likely to be viewed face-on and, hence, the viewing angle would be down the magnetic field lines. No significant circular polarisation was found, with $3 \sigma$ upper limits of $0.48,0.96$ and $0.69 \%$ in the $U, B$, and $V$-bands, respectively.

Although polars possess strong variable soft X-ray fluxes, they also tend to show harder X-rays, produced by the shocked accretion column (Cropper 1990). In the present case of HV Cet, no strong hard X-ray emission is detected.

\section{Discussion}

Swift monitoring campaigns have shown that novae can have very different X-ray-UV relationships. Some, such as V2491 Cyg (Page et al. 2010) show little or no correlation between the bands, implying differences in the emission mechanisms; V458 Vul, on the other hand, shows an approximate anticorrelation between the X-ray and UV data (Drake et al. 2008), which might be explained by a single process which is changing in characteristic energy. Additionally, the in-phase variation of the X-rays and UV observed in HV Cet implies a single mechanism (see also Schwarz et al. 2011), which changes in luminosity, though not (strongly) in temperature (see Sect. 2.2 for X-ray spectral fits).

We take the $1.77 \mathrm{~d}$ modulation seen in both the X-ray and UV data to be the orbital period of the system. It is unlikely to be the WD spin period as accretion will have spun it up to much shorter periods, although not up to break-up velocities. Typical rotational velocities for such accreting WDs are of the order $v \sin i \sim 200-400 \mathrm{~km} \mathrm{~s}^{-1}$ (e.g., Szkody et al. 2002; Starrfield et al. 2004), corresponding to a spin period of $\sim 1 \mathrm{~min}$. Disc precession could possibly occur in this system due to the strong illumination or other mechanisms (Sood et al. 2007; Farrell et al. 2009), but the observed period is shorter than other known superorbital examples, and then requires a much shorter binary period.
It is possible that the $40-50$ day modulation of the light-curve could originate in this way.

An orbital period of $1.77 \mathrm{~d}$ would be very long for a polar, more than five times that of the current secure longest period system V1309 Ori $\left(P_{\text {orb }}=0.333\right.$ day, Staude et al. 2001; typical polar orbital periods are measured in hours, e.g. Warner 1995). Synchronisation arguments imply an extremely high WD magnetic field $\left(B_{\mathrm{WD}, \mathrm{min}} \sim P_{\mathrm{orb}}^{7 / 3}\right.$; Page et al. 2010). In this case, the lack of hard X-ray emission is not such a strong reason to reject the polar idea, because, with a high field, optical cyclotron emission is significant in reducing the shock-induced Bremsstrahlung X-ray emission (Lamb \& Masters 1979; see Cropper et al. 1999, for a calculated example). In addition, a high magnetic field also tends to suppress optical polarisation, displacing it to the UV (e.g. Cropper et al. 1989). Nevertheless, the very long period and the absence of both hard X-rays and optical polarised flux leads us not to favour a polar origin for the $1.77 \mathrm{~d}$ modulation of the light curve. It is interesting to note that a polar origin of the light-curve of Nova Mus 1983 (GQ Mus, $P_{\text {orb }}=85 \mathrm{~min}$ ) has been strongly argued by Diaz \& Steiner (1994).

Although this $1.77 \mathrm{~d}$ period is longer than that of many novae, it is not unprecedented; the Ritter \& Kolb (2003) CV catalogue (June 2011 ed. 7.16) lists four novae with orbital periods equal to or longer than this. Persistent SSS have orbital periods similar to that of HV Cet; examples include RX J0019.8+2156 (0.66 day; Beuermann et al. 1995), RX J0513.9-6951 (0.74 day; Motch \& Pakull 1996), CAL 83 (1.04 day; Smale et al. 1988) and CAL 87 (0.44 day; Callanan et al. 1989). Indeed, CAL 87 shows small and variable secondary dips during the peaks of its optical modulation as seen in the UV data for HV Cet (Figs. 1 and 4). It has been suggested that these secondary minima could be explained by the eclipsing of a bright accretion disc (Kahabka \& van den Heuvel 1997), while Schandl et al. (1997) present a model which suggests that the dip is produced by the obscuration of the secondary star by a disc with variable rim height.

By day 300 no X-ray source was detected at the position of $\mathrm{HV}$ Cet (to a limit of $<2 \times 10^{-3}$ count $^{-1}$ ) and, similarly, no source was detected in the ROSAT All Sky Survey to an XRT equivalent rate of $1.6 \times 10^{-4}$ count s$^{-1}$, indicating $\mathrm{HV}$ Cet is 
not a persistent SSS. While most CBSSs are persistent X-ray sources, at least one has varied by more than a factor of 1000 (RX J0513.9-6951; Schaeidt et al. 1993). HV Cet shares other properties with the CBSS population, too. The presence of jets is inferred in both HV Cet (Pejcha et al. 2008) and CBSSs (Cowley et al. 1998) by the tri-peaked emission lines, thus implying a well-formed accretion disc (Livio 1999). In addition, it is striking that both HV Cet and CBSSs have strong orbital modulation which varies in phase between the optical and the $\mathrm{X}$-ray. In the case of most CBSSs, an accretion disc rim, highest where the stream from the secondary impacts (up to $z / r=0.4$; Meyer-Hofmeister et al. 1997), occults both its own bright inner surface and the bright inner region of the disc. This inner region is expected to scatter X-rays from the central hot WD (Kahabka 1996; Crampton et al. 1997; Schandl et al. 1997; Meyer-Hofmeister et al. 1997). A similar inner X-ray scattering volume was proposed recently for the nova U Sco (Ness et al. 2012). Of course, a system with both disc rim occultations and high velocity jets requires a particular intermediate inclination. An alternative to a jet interpretation of distinct high velocity line components is to ascribe them to a high inclination accretion disc (cf. Horne \& Marsh 1986). A high binary inclination is a natural inference of the large photometric periodic modulation seen, and should be verifiable by spectroscopy in quiescence.

The UV flux seen by GALEX and the UVOT is a factor 1001000 times the flux expected from a blackbody or model atmosphere fitted to the X-ray spectrum (the large range comes from the $90 \%$ confidence temperature uncertainty of the fits). We propose that the X-ray flux that we see is the result of optically thin electron scattering by hot gas, substantially obscured by the accretion disc rim which permanently hides the WD itself. Our inferred luminosity of $\sim 100 L_{\text {Sun }}$ (Fig. 5) is a factor $\sim 300$ less than the expected Eddington luminosity, suggestive of substantial obscuration. Correction for this effect results in a more reasonable white dwarf photospheric radius of $\sim 0.5 \times 10^{9} \mathrm{~cm}$. The significant extent of the scattering cloud accounts for the smooth and broad X-ray modulation at the $1.77 \mathrm{~d}$ period, the modulation being due to the orbital motion of the higher disc rim in the region of the impact point of the gas stream from the secondary star. The UV flux is proposed to be reprocessed X-rays; the inner side of the disc-stream impact region sees the full luminosity of the WD due to the optically thin nature of the scattering region, while we observe a UV maximum at the phase of X-ray maximum due to the reprocessing disc-stream interior surface having maximum aspect at the time of minimum X-ray obscuration. The broad UV modulation naturally follows from the changing projected area of the reprocessing site. The dense gas in the outer accretion disc is not likely effectively to reflect soft X-rays however, as photoelectric absorption would give this process a very low efficiency. The presence of the $1.77 \mathrm{~d}$ modulation on day 65 clearly implies the existence of a well-developed accretion disc at this time, consistent with the disc-reformation witnessed in $\mathrm{U}$ Sco some 23-35 days after outburst (Ness et al. 2012). Accretion onto the WD is therefore likely to be occurring, potentially feeding the nuclear burning and extending the life of the super-soft $\mathrm{X}$-ray source.

The first high-cadence set of Swift observations of HV Cet, taken on days 65 to 80 , show a striking pattern in which the $\mathrm{X}$-ray maxima decline while the minima remain approximately constant, yet over the same interval the UV minima decline strongly, whilst the maxima vary only slightly. The optical, UV and X-ray flux modulations are in phase, suggesting X-ray reprocessing as an origin of the UV/optical flux. If the modulation is due to eclipses then they are partial and do not vary in width in any observed band. While this pattern cries out for an explanation, it does not appear possible to generate this behaviour from a simple occulting structured disc-rim model by individual variation of either the disc rim height or optical depth, by variation of the hot spot size, or by variation of the inner disc scattering region size or brightness.

\section{Summary}

We have extended the original discovery work on the optical/X-ray transient CSS 081007 of Beardmore et al. (2010) by the inclusion of optical photometry, GALEX UV spectra, time-resolved WD model atmosphere X-ray spectral fits and consideration of the X-ray/UV/optical pulse profile evolution. We show that the $1.77 \mathrm{~d}$ periodic flux modulation, seen to vary in phase in all three wavebands, is most likely to be orbital. Making use of compact binary supersoft source ideas, we suspect that disc rim occultation of an inner scattering region permanently hides the WD, and the disc-stream interaction region is the cause of the periodic X-ray modulation, while the UV flux is due to X-ray reprocessing in its interior surface, even if simple model concepts do not explain some of the striking changes seen. A synchronous magnetic WD was rejected partly because of the lack of hard X-rays and optical polarisation detections, but mostly because the long period requires a magnetic field on the white dwarf very much larger than any detected so far.

Although it is likely that the optically brightest part of the outburst was missed, making the outburst date quite uncertain, HV Cet appears to be a fast Ne nova at an unusually high Galactic latitude. It had a supersoft X-ray phase lasting at least 120 days. UV flux peaks separated by $\sim 40$ days were matched to a lesser extent in the X-ray light curve; we speculate that this might represent a super-orbital period. A high expansion velocity at peak, and thus a high mass WD, is clearly implied by rapid X-ray turn-off (Schwarz et al. 2011). Neither period is associated with soft X-ray spectral changes, which are restricted to a rise from $\sim 60$ to $\sim 80 \mathrm{eV}$ between 110 and 130 days after discovery and subsequent decline at the end of the supersoft phase around day 150 . The X-ray luminosity derived from the model atmosphere fits is sub-Eddington, but is still sufficient to power the re-emitted UV flux, consistent with our ideas on the formation of the periodic light curve.

Many aspects of this enigmatic source remain to be understood and we hope that optical spectroscopy will clarify the nature of this system. The coming age of large area transient surveys, ideally matched by similar surveys at X-ray energies, can be expected to find large numbers of systems such as this one.

Acknowledgements. A.P.B., J.P.O., K.L.P. and P.A.E. acknowledge support by the UK Space Agency. We thank Graham Wynn and Mike Bode for useful discussions, and the Swift PI, Neil Gehrels, and the science and mission operations teams for their support of these observations. We acknowledge with thanks the variable star observations from the AAVSO International Database contributed by observers worldwide and used in this research and thank Klaas Wiersema for assistance with the reduction of the Dutch photometry. S.S. acknowledges partial support from NSF and NASA grants to ASU. This work made use of data supplied by the UK Swift Science Data Centre at the University of Leicester. We thank the referee for comments which resulted in improvement to the paper.

\section{References}

Beardmore, A. P., Osborne, J. P., Page, K., et al. 2008, The Astronomer's Telegram, 1873

Beardmore, A. P., Osborne, J. P., Page, K. L., et al. 2010a, AN, 331, 156 Beardmore, A. P., Balman, S., Osborne, J. P., et al. 2010b, The Astronomer's Telegram, 2423 
Beuermann, K, Reinsch, K., Barwig, H, et al. 1995, A\&A, 294, L1

Bode, M. F., \& Evans, A. 2008, Classical Novae, 2nd edn. (Cambridge: CUP) Astrophysics Series, 43

Bode, M. F., Osborne, J. P., Page, K. L., et al. 2009a, The Astronomer's Telegram, 2001

Bode, M. F., Osborne, J. P., Page, K. L., et al. 2009b, The Astronomer's Telegram, 2025

Bode, M. F., Osborne, J. P., Page, K. L., et al. 2010, The Astronomer's Telegram, 2392

Burrows, D. N., Hill, J. E., Nousek, J. A., et al. 2005, Space Sci. Rev., 120, 165

Callanan, P. J., Machin, G., Naylor, T., \& Charles, P. A. 1989, MNRAS, 241, 37

Cowley, A. P., Schmidtke, P. C., Crampton, D., \& Hutchings, J. B. 1998, ApJ, 504,854

Crampton, D., Hutchings, J. B., Cowley, A. P., \& Schmidtke, P. C. 1997, ApJ, 489, 903

Cropper, M. 1990, SSRv, 54, 195

Cropper, M., Mason, K. O., Allington-Smith, J. R., et al. 1989, MNRAS, 236, $29 \mathrm{p}$

Cropper, M., Wu, K., Ramsay, G., \& Kocabiyik, A. 1999, MNRAS, 306, 684

Diaz, M. P., \& Steiner, J. E. 1994, ApJ, 425, 252

Di Stefano, R., Primini, F. A., Liu, J., Kong, A., \& Patel, B. 2010, AN, 331, 205

Drake, J. J., Wagner, R. M., Starrfield, S., et al. 2003, ApJ, 584, 448

Drake, J. J., Page, K. L., Osborne, J. P., et al. 2008, The Astronomer's Telegram, 1721

Drake, A. J., Djorgovski, S. G., Mahabal, A., et al. 2009a, ApJ, 696, 870

Drake, A. J., Beshore, E. C., Djorgovski, S. G., et al. 2009b, The Astronomer's Telegram, 1940, 20

Evans, P. A., Beardmore, A. P., Page, K. L., et al. 2009, MNRAS, 397, 1177

Farrell, S. A., Barret, D., \& Skinner, G. K. 2009, MNRAS, 393, 139

Ferland, G. J., Korista, K. T., Verner, D. A., et al. 1998, PASP, 110, 761

Gallagher, J. S., III, \& Code, A. D. 1974, ApJ, 189, 303

Gehrels, N., Chincarini, G., Giommi, P., et al. 2004, ApJ, 611, 1005

Goad, M. R., Tyler, L. G., Beardmore, A. P., et al. 2007, A\&A, 476, 1401

Hearn, D. R., \& Richardson, J. A. 1977, ApJ, 213, L115

Hellier, C. 2001, Cataclysmic Variable Stars: How and Why they Vary (SpringerPraxis Publishing Ltd.)

Horne, K., \& Marsh, T. R. 1986, MNRAS, 218, 761

Iben, I., Jr 1982, 259, 244

Kahabka, P. 1996, A\&A, 306, 795

Kahabka, P., \& van den Heuvel, E. P. J. 1997, ARA\&A, 35, 69

Kazarovets, E. V., Samus, N. N., Durlevich, O. V., Kireeva, N. N., \& Pastukhova, E. N. 2011, IBVS, 5969

Krautter, J., Ögelman, H., Starrfield, S., Wichmann, R., \& Pfeffermann, E. 1996, ApJ, 456, 788

Lamb, D. Q., \& Masters, A. R. 1979, A\&A, 234, L117

Livio, M. 1999, Phys. Rep., 311, 225

MacDonald, J., Fujimoto, Y., \& Truran, J. W. 1985, ApJ, 294, 263

Meyer-Hofmeister, E., Schandl, S., \& Meyer, F. 1997, A\&A, 321, 245

Morrissey, P., Conrow, T., Barlow, T. A., et al. 2007, ApJS, 173, 682

Motch, C., \& Pakull, M. W. 1996, Lect. Notes Phys., 472, 127

Nelson, T., \& Orio, M. 2009, The Astronomer's Telegram, 1910

Ness, J.-U., Starrfield, S., Burwitz, V., et al. 2003, ApJ, 594, L127

Ness, J.-U., Schaefer, B.E., Dobrotka, A., et al. 2012, ApJ, 745, 43
Orio, M., Parmar, A. N., Greiner, J., et al. 2002, MNRAS, 333, L11

Osborne, J. P., Beardmore, A. P., Page, K. L., et al. 2009, The Astronomer's Telegram, 1942

Osborne, J. P., Page, K. L., Beardmore, A. P., et al. 2011, ApJ, 727, 124

Page, K. L., Osborne, J. P., Evans, P. A., et al. 2010, MNRAS, 401, 121

Page, K. L., Walter, F. M., Schwarz, G. J., et al. 2012, The Astronomer's Telegram, 4043

Pei, Y. C. 1992, ApJ, 395, 130

Pejcha, O., Prieto, J. L., \& Denney, K. 2008, Astronomer's Telegram, 1825

Pojmanski, G. 2002, Acta Astron., 52, 397

Poole, T. S., Breeveld, A. A., Page, M. J., et al. 2008, MNRAS, 383, 627

Porter, R. L., Ferland, G. J., Kraemer, S. B., et al. 2006, PASP, 118, 920

Prialnik, D. 1986, ApJ, 310, 222

Prieto, J. L., Denney, K., Pejcha, O., \& Wagner, R. M. 2008, The Astronomer's Telegram, 1835

Rauch, T. 2003, A\&A, 403, 709

Rauch, T., Orio, M., Gonzales-Riestra, R., et al. 2010, ApJ, 717, 363

Ritter, H., \& Kolb, U. 2003, A\&A, 404, 301

Roming, P. W. A., Kennedy, T. E., Mason, K. O., et al. 2005, Space Sci. Rev., 120,95

Schaefer, B. E. 2010, ApJS, 187, 275

Schaeidt, S., Hasinger, G., \& Trümper, J. 1993, A\&A, 270, L9

Schandl, S., Meyer-Hofmeister, E., \& Meyer, F. 1997, A\&A, 318, 73

Schlegel, D. J., Finkbeiner, D. P., \& Davis, M. 1998, ApJ, 500, 525

Schwarz, G. J. 2002, ApJ, 577, 940

Schwarz, G. J., Ness, J.-U., Osborne, J. P., et al. 2011, ApJS, 197, 31

Schwarz, G. J., Ness, J.-U., Osborne, J. P., et al. 2008, The Astronomer's Telegram, 1847

Schwarz, G. J., Shore, S. N., Starrfield, S., \& Vanlandingham, K. M. 2007, ApJ, 657,453

Schwarz, G. J., Starrfield, S., Shore, S. N., \& Hauschildt, P. H. 1997, MNRAS, 290, 75

Smale, A. P., Corbet, R. H. D., Charles, P. A., et al. 1988, MNRAS, 233, 51

Sood, R., Farrell, S., O’Neil, P., \& Dieters, S. 2007, Adv. Space Res., 40, 1528

Sparks, W. M., Starrfield, S., \& Truran, J.W. 1976, ApJ, 208, 819

Starrfield, S., Sion, E. M., \& Szkody, P. 2004, eds. A. Maeder, \& P. Eenens, Proc. IAU Symp., 215, 551

Starrfield, S., Timmes, F. X., Iliadis, C., et al. 2012, Baltic Astron., 21, 76

Staude, A., Schwope, A. D., \& Schwarz, R. 2001, 374, 588

Sterken, C. 2005, in The Light-Time Effect in Astrophysics, ed. C. Sterken, ASP Conf. Ser., 335, 3

Stockman, H. S., Schmidt, G. D., \& Lamb, D. Q. 1988, ApJ, 332, 282

Stockman, H. S., Schmidt, G. D., Angel, J. R. P., et al. 1977, ApJ, 217, 815

Szkody, P., Sion, E. M., Gänsicke, B. T., \& Howell, S. B. 2002, in The Physics of Cataclysmic Variables and Related Objects, ed. B. T. Gänsicke, \& K. Beuermann, Reinsch, ASP Conf. Proc., 261, 21

Templeton, M., Koff, R., Wils, P., \& Henden, A. 2010, JAAVSO, 38, 147

Trümper, J., Hasinger, G., Aschenbach, B., et al. 1991, Nature, 349, 579

van den Heuvel, E. P. J., Bhattacharya, D., Nomoto, K., \& Rappaport, S. A. 1992, A\&A, 262, 97

Warner, B. 1995, Cataclysmic variable stars, Cambridge Astrophys. Ser., 28

Williams, R. E., Phillips, M. M., \& Hamuy, M. 1994, ApJS, 90, 297 